\title{
The management of depression during pregnancy: a report from the American Psychiatric Association and the American College of Obstetricians and Gynecologists म $^{4}$
}

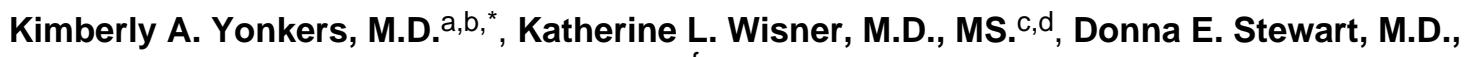 \\ FRCPC $^{e}$, Tim F. Oberlander, M.D., FRCPC ${ }^{f}$, Diana L. Dell, M.D., FACOG ${ }^{g}$, Nada Stotland, \\ M.D., M.P.H. ${ }^{\text {h,i }}$, Susan Ramin, M.D., FACOGj, Linda Chaudron, M.D., MS. ${ }^{k}$, and Charles \\ Lockwood, M.D., FACOG' \\ aDepartment of Psychiatry, Epidemiology and Public Health, Yale School of Medicine, New \\ Haven, CT 06510, USA \\ ${ }^{b}$ Department of Obstetrics and Gynecology and Reproductive Sciences, Yale School of Medicine, \\ New Haven, CT 06510, USA \\ 'Department of Psychiatry, Obstetrics and Gynecology, Women's Behavioral, HealthCARE, \\ Western Psychiatric Institute and Clinic, University of Pittsburgh Medical Center, Pittsburgh, PA \\ 15213, USA \\ dDepartment of Reproductive Sciences, Epidemiology and Women's Studies, Women's \\ Behavioral, HealthCARE, Western Psychiatric Institute and Clinic, University of Pittsburgh \\ Medical Center, Pittsburgh, PA 15213, USA \\ eDepartments of Psychiatry, Obstetrics and Gynecology, Family and Community Medicine, \\ Medicine, Surgery and Anesthesia, University Health Network Women's Health Program, \\ University of Toronto, Toronto, Ontario, Canada M5G 2C4 \\ fDepartment of Pediatrics, University of British Columbia, Human Early Experience Unit, Centre \\ for Community Child Health Research, Child and Family Research Institute, Vancouver, British \\ Columbia, Canada V6 3V4 \\ gDuke University Medical Center, DUMC Box 3263, Durham, NC 27710, USA \\ hDepartment of Psychiatry and Obstetrics, Rush Medical College Chicago, IL 60637, USA \\ 'Department of Gynecology, Rush Medical College Chicago, IL 60637, USA \\ iThe University of Texas Health Science Center at Houston, Department of Obstetrics, \\ Gynecology, and Reproductive Sciences, Division of Maternal-Fetal Medicine, Houston, TX \\ 77030, USA \\ kDepartment of Psychiatry, Pediatrics and Obstetrics and Gynecology, University of Rochester, \\ Rochester, NY 14642, USA
}

\footnotetext{
${ }^{2}$ Dr. Yonkers has received a research grant from Eli Lilly this past year. She has received study drug from Pfizer for a National Institute of Mental Health trial. Dr. Stewart serves on the Advisory Boards ofWyeth and Eli Lilly and Boehringer Ingelheim. Dr. Dell has received research grants from Pfizer has consulted to Bayer Schering Pharma AG and Berlex and is on the Speaker's Bureau for Berlex, GlaxoSmithKline, Pfizer and Wyeth. Dr. Wisner serves on the Speaker's Board of Glaxo Smith Klein. Dr. Oberlander; Dr. Ramin; and Drs. Stotland, Chaudron, and Lockwood have no conflicts to disclose.

(C) 2009 American Psychiatric Association. Published by Elsevier Inc. All rights reserved.

*Corresponding author. Department of Psychiatry, Epidemiology and Public Health, Yale School of Medicine, New Haven, CT, USA. Fax: +1 203764 6766. kimberly.yonkers@yale.edu (K.A. Yonkers).
} 
'Department of Obstetrics, Gynecology and Reproductive Sciences, Yale University School of Medicine, PO Box 208063, New Haven, CT 06520-8063, USA

\section{Abstract}

Objective-To address the maternal and neonatal risks of both depression and antidepressant exposure and develop algorithms for periconceptional and antenatal management.

Method-Representatives from the American Psychiatric Association, the American College of Obstetricians and Gynecologists and a consulting developmental pediatrician collaborated to review English language articles on fetal and neonatal outcomes associated with depression and antidepressant treatment during childbearing. Articles were obtained from Medline searches and bibliographies. Search keywords included pregnancy, pregnancy complications, pregnancy outcomes, depressive disorder, depressive disorder/dt, abnormalities/drug-induced/epidemiology, abnormalities/drug-induced/et. Iterative draft manuscripts were reviewed until consensus was achieved.

Results-Both depressive symptoms and antidepressant exposure are associated with fetal growth changes and shorter gestations, but the majority of studies that evaluated antidepressant risks were unable to control for the possible effects of a depressive disorder. Short-term neonatal irritability and neurobehavioral changes are also linked with maternal depression and antidepressant treatment. Several studies report fetal malformations in association with first trimester antidepressant exposure but there is no specific pattern of defects for individual medications or class of agents. The association between paroxetine and cardiac defects is more often found in studies that included all malformations rather than clinically significant malformations. Late gestational use of selective serotonin reuptake inhibitor antidepressants is associated with transitory neonatal signs and a low risk for persistent pulmonary hypertension in the newborn. Psychotherapy alone is an appropriate treatment for some pregnant women; however, others prefer pharmacotherapy or may require pharmacological treatment.

Conclusions-Antidepressant use in pregnancy is well studied, but available research has not yet adequately controlled for other factors that may influence birth outcomes including maternal illness or problematic health behaviors that can adversely affect pregnancy.

\section{Keywords}

Depression; Pregnancy; Antidepressant agents

\section{Introduction}

Between $14 \%$ and $23 \%$ of pregnant women will experience a depressive disorder while pregnant [1]. In 2003, approximately $13 \%$ of women took an antidepressant at some point in pregnancy, a rate that has doubled since 1999 [2]. Thus, clinicians and patients need up-todate information to assist with decisions about depression treatment during pregnancy. To meet this need, the American Psychiatric Association and the American College of Obstetricians and Gynecologists convened a work group to critically evaluate and summarize information about the risks associated with depression and antidepressant treatment during pregnancy. The group was comprised of clinical research experts within these two medical specialties and a developmental pediatrician. By consensus, the authors selected studies that were methodologically sound as evidenced by clearly stated variables and sample sizes that were appropriate to test stated hypotheses. This report is published jointly in a psychiatric and an obstetrical journal to ensure that clinicians have easy access to the information. 
Readers should consider several limitations when interpreting the literature. Only a minority of reports that assessed associations between antidepressant treatment and birth outcomes included information on maternal psychiatric illness. When available, such information was typically in the form of clinicians' reports from administrative databases or self-report screening measures. The studies with detailed information regarding diagnoses and antidepressant use were usually smaller and had limited power to find important associations. Finally, confounding factors that influence birth outcomes, such as poor prenatal care and drug, alcohol and nicotine use were variably controlled. These potential confounders occur at a higher rate in depressed compared to nondepressed women [3] Similarly, pregnancy complications such as nausea, vomiting, hyperemesis gravidarum, and pre-eclampsia occur at a higher rate in depressed compared to non-depressed women [4].

The convergence of results from studies with different designs was a factor used in this report to evaluate the validity of associations between antidepressant exposures and adverse reproductive outcomes. We review findings that refer to associations between maternal depression and use of antidepressants with regard to specific perinatal outcomes. At the end of the report, we present strategies for the management of women with depression who are either pregnant or planning a pregnancy.

\section{Maternal depression and adverse reproductive outcomes}

\subsection{Miscarriage}

Reasons for miscarriage, especially early events, are difficult to obtain because prospective data collection must begin with pregnancy recognition. Fetal loss after recognition of pregnancy, typically after 6 weeks gestation, only occurs in about $8 \%$ of women and large sample sizes are needed to assess differences among exposures associated with this outcome. Accordingly, there is a paucity of information about depression and spontaneous pregnancy loss. We found existing studies problematic because of small sample sizes and other methodological limitations, which preclude definitive conclusions [5-7].

\subsection{Growth effects}

Fetal growth effects are typically manifested as delivery of low birth weight (LBW) (born $<2500 \mathrm{~g}$ ) or small for gestational age (SGA) (typically birth weight $<10 \%$ of age-adjusted weight) infants. Depressive symptoms in the mother have been linked with an increased risk for delivery of a LBW [8] or SGA infant [9] in some but not all studies [10,11]. Only the latter negative reports included structured diagnostic assessments for depression but the numbers of women with major depressive disorder (MDD) were small in both studies. The current state of information does not support or refute an association between MDD and LBW or SGA delivery.

\subsection{Preterm delivery}

Preterm deliveries (PTD) are deliveries that occur prior to 37 completed weeks of gestation. Several studies report an association between depressive symptoms or a depressive disorder and shorter gestations, including PTD [8,9,12], although findings are not consistent [10,11]. The same limitations discussed above apply to the outcomes of PTD and gestational age, and thus, available data neither support nor refute a link between MDD and these outcomes.

\subsection{Neonatal effects}

We could identify no studies that link maternal depression to congenital anomalies in their infants. However, neonates born to mothers with a depressive disorder have increased risk for irritability, less activity and attentiveness, and fewer facial expressions compared to offspring born to mothers without depression [13]. This suggests that either genetic and/or 
other perinatal factors have an impact on infant behavior. In small studies, newborns of depressed women have physiologic profiles that parallel those of their mothers and include elevated cortisol, decreased peripheral levels of dopamine and serotonin, greater relative right frontal electroencephalogram activation and lower vagal tone [13].

\subsection{Long-term effects on offspring}

Surprisingly, little research has looked at the specific effects of antenatal depression on late neonatal or post neonatal development in children although maternal depression after delivery has received extensive attention. In general, depressive symptoms in the mother, rather than a diagnosis of a maternal depressive disorder, has been the exposure of interest in the few existing studies [14-16]. One large cohort study attempted to assess the specific effects of depressive symptoms, according to the Edinburgh Postnatal Depression Scale (EPDS), in mothers during pregnancy although child performance was based upon maternal report only and potential bias cannot be excluded [16]. In this study, children exposed to maternal depressive symptoms at 18 and 32 weeks gestation, but not postnatally, showed greater developmental delay at 18 months than children with mothers who were not depressed in pregnancy. The odds ratio (OR) for developmental problems for offspring born to a mother with depressive symptoms according to a cutoff of 12 on the EPDS was 1.34 (95\% CI=1.01-1.78; $P=.043$ ). The only study to use independent assessors found no effect of depression in pregnancy on child development [14], while the third study used maternal report of child development and found possible indirect effects of antenatal depression, particularly for couples who required infertility treatment [15]. The possibility of bias and existence of studies that do not agree support the need for more work to determine the degree of later developmental risk conferred to a child when mother is depressed in pregnancy.

\section{The impact of antidepressants on birth outcomes}

More than $80 \%$ of women take at least one dose of a medication, exclusive of vitamins, during pregnancy [17]. The use of multiple medications during pregnancy makes it difficult to assess the impact of a single compound, such as an antidepressant, on maternal and fetal outcomes. Unfortunately, women who are undergoing treatment with an antidepressant agent are more likely to take another prescription medication [18]. Selective serotonin reuptake inhibitors (SSRIs) are the most frequently prescribed class; clinicians infrequently use tricyclic antidepressants (TCAs) and monoamine oxidase inhibitors in contemporary practice. Current antidepressant treatment usage among pregnant patients follows this prescription pattern [2].

\subsection{Miscarriage}

Increased risk for spontaneous abortion is associated with the use of various antidepressants in early pregnancy [19-24]. In a quantitative review $(n=3567)$, miscarriage rates were $12.4 \%$ for women who used antidepressants versus $8.7 \%$ for unexposed women (RR=1.45; $95 \%$ $\mathrm{CI}=1.19-1.77$ ) [25]. No differences were observed among the various classes of antidepressants. This investigation predominantly included late miscarriage since rates in both groups were low compared to population-based rates [26]. None of the studies that contributed data controlled for psychiatric illness state. Moreover, confounding health habits, including smoking and drug use, and age were variably controlled among studies that contributed to the meta-analysis. Studies with adequate control for potential confounding variables, especially age, which is a strong predictor of spontaneous abortion, are needed before an association can be established. 


\subsection{Growth effects}

Reductions in birth weight, LBW and SGA, are associated with SSRI use in pregnancy $[9,19,27,28]$. Not all studies show this association [20-22,24,29,30], although only a few had adequate power to find a difference [31,9]. An analysis from a Swedish birth registry found that LBW deliveries were associated with both SSRI and TCA use; however, the infants were not SGA, which suggests that the elevated LBW rates were attributable to early births of babies that had appropriate weight for their gestational age [32].

Several authors attempted to "control" for the possible effects of maternal illness by inclusion of women with a depressive disorder who were or were not treated with SSRIs $[11,27]$ or by propensity score matching $[9,33]$. The effect of SSRIs on birth weight persists in these studies after statistical control of possible confounders, which supports a true association between maternal SSRI use and reduced infant birth weight. After application of propensity score matching, which attempts to equalize variables among the treated and untreated depressed women, rates of SGA infants in the SSRI exposed group were significantly higher than in women who were depressed and not treated with antidepressants, but the absolute difference in incidence risk for this outcome was small and is estimated to be only $0.033(95 \% \mathrm{CI}=0.007-0.059)$ [9].

The time and duration of exposure to an antidepressant are additional factors that are difficult to disentangle. In many instances, third trimester exposure will occur among women who took medication throughout pregnancy rather than third trimester only. This contrasts with first trimester exposure, which, by definition, only occurred in the first trimester and hence is briefer. A third consideration is the likelihood that women who require medication during the third trimester may have more severe underlying illnesses. It is not possible to say whether the time point at which an exposure occurred, a longer duration of exposure or more severe illness confers the greater impact on growth [33].

\subsection{Gestational age effects}

The rate of preterm birth ( $<37$ weeks gestation) in the United States is $12.7 \%$ and is the leading cause of perinatal morbidity and mortality and hence the relationship between antidepressant use and this outcome requires urgent clarification [34]. Evidence that PTD ( $<37$ completed weeks of pregnancy) is significantly higher among women who used antidepressants, including SSRIs and TCAs, has been found by numerous groups $[19,27,29,32,35]$. Other studies do not support an association [24,30,31]. Studies that find an effect for antidepressants on gestational age typically find modest differences in mean gestational duration of one week or less for those exposed compared to those not exposed. [22,27,30]. Application of propensity scores to control for confounders eliminated differences in the rates of PTD among women who did or did not take an antidepressant agent [9]. Further studies showed that the effects of SSRIs on gestational age are dependent on the duration of in utero antidepressant exposure and that longer exposures are more likely to decrease gestational age [33].

\subsection{Structural malformations}

3.4.1. Tricyclic antidepressants-The majority of studies have not shown an association between TCA use in pregnancy and structural malformations $[27,36]$.

3.4.2. Serotonin reuptake inhibitors-The majority of linked database [18,31] and two recent case cohort studies $[37,38]$ found no increased rate of major or specific cardiac malformations when in utero SSRI exposure was considered in aggregate. While some linked database reports find that compared to unexposed offspring, those exposed to paroxetine during the first trimester are at higher risk of cardiac malformations $[18,39,40]$, 
these results are disputed by other reports including several large case cohort studies $[37,38]$. Another group failed to find an association between maternal paroxetine use and cardiac defects in a study that included 1170 women who took paroxetine early in pregnancy [41]. However, they did find a difference between their risk estimates derived from teratogen information services and those derived from database studies. The tendency of linked database studies to report all malformations and include those that are clinically insignificant may account for differences. Specific defects found by some groups include ventricular outflow defects, craniosynotosis and omphalocele [37,38]. Again, these risks are extremely small and not replicated by other studies [18].

Infants exposed in utero to an SSRI in combination with a benzodiazepine but not an SSRI alone, may have a higher a incidence of congenital heart defects compared to no exposure, even after controlling for maternal illness characteristics [42]. Such results raise the possibility that presumed associations between antidepressants and malformations may be complicated by poly-drug interactions.

To summarize, the current data on SSRI exposure show no consistent information to support specific morphological teratogenic risks. Concurrent medication use or health habits confound possible associations and create methodological challenges in this area of investigation.

3.4.3. Other antidepressants-Other antidepressants include bupropion (Wellbutrin and Zyban), venlafaxine (Effexor), duloxetine (Cymbalta), nefazodone, and mirtazepine (Remeron). These agents vary in chemical structure and putative mechanism of action. None of the studies found a statistically significant difference or higher than expected rate of congenital anomalies when compared to controls who were either taking other antidepressants [40] or taking agents known not to be teratogenic; however, data are more sparse than for the SSRIs or TCAs [20,21,29,39]. Using the Swedish Medical Birth Registry, investigators identified 732 pregnant women who used SNRI/NRI drugs in early pregnancy and compared their outcomes to all women in the population. The rate of PTD was significantly increased $(\mathrm{OR}=1.6 ; 95 \% \mathrm{CI}=1.19-2.15)$, and neonatal symptoms (respiratory problems, low Apgar score, hypoglycemia, and neonatal convulsions) showed a pattern similar to that observed after maternal SSRI treatment. No increased risk for stillbirths or congenital malformations was observed [43].

\subsection{Neonatal neurobehavioral outcomes}

3.5.1. Tricyclic antidepressants-In utero exposure to TCAs are associated with increased perinatal complications including jitteriness, irritability and, rarely, convulsions in neonates $[32,36]$.

3.5.2. Serotonin reuptake inhibitors-A cluster of symptoms termed "poor neonatal adaptation" [44] has been reported during the immediate neonatal days among infants exposed to SSRIs. Symptoms include tachypnea, hypoglycemia, temperature instability, irritability, a weak or absent cry, and seizures [19,35]. These symptoms occurred in 15-30\% of women who took SSRIs in late pregnancy $[19,33,35]$. Symptoms in neonates were transient and typically resolved by 2 weeks or sooner after delivery. While the underlying mechanisms remain to be determined, a number of hypotheses have been proposed to account for these symptoms, including a gene-SSRI interaction [45], a transient increase and /or suppression of monoamine neurotransmitters [46] reflecting a "withdrawal" or "discontinuation" syndrome, pharmacological toxicity related to increased SSRI levels [47] or sustained changes in brain function [48]. 


\subsection{Other perinatal complications}

Other perinatal complications have been reported among women who used antidepressants in late pregnancy. An increased risk of persistent pulmonary hypertension (PPHN) was found among newborns whose mothers were treated SSRIs with a greater risk for infants who were exposed later in pregnancy $[44,49]$. PPHN is characterized by right to left shunting of blood through the ductus arteriosus and foramen ovale and results in neonatal hypoxia. If this is chronic and severe, babies can develop right heart failure. The base rate of this condition is between 0.5 and 2 per thousand and is fatal in about $10 \%$ of cases [50]. Recent studies suggest that the absolute risk may be elevated to 3-6 per 1000 among infants exposed to SSRIs in pregnancy $[19,49]$. Exposure to non-SSRI antidepressants in these studies were not associated with PPHN [19], but tests of such associations are limited by the less frequent use of other classes of medication in pregnancy. Respiratory distress in the newborn may occur along a spectrum of seriousness in association with maternal SSRI use with PPHN among the most serious consequences [19]. Women who have longer rather than shorter periods of SSRI treatment may be at higher risk of delivery of an infant with respiratory complications [33].

\subsection{Long-term effects on offspring}

Limited information is available about possible long-term effects of SSRI exposure in utero. Adverse neurocognitive effects at 6-9 months after delivery were not associated with maternal SSRI use in one small study [51]. In another report from the same study, the use of TCAs in pregnancy was not linked with differences in general behavior, cognitive function, and temperament in young children [52].

More work is needed to distinguish the specific effects of prenatal psychotropic medication exposure from the effects of antenatal and postnatal depression. It should be stressed that there may be developmental risks for children with depressed and anxious parents independent of psychotropic drug exposure. Careful follow-up of early development is essential as this may identify "red flags" to subsequent emotional and academic problems later in childhood. Particular attention should be paid to regulatory problems (e.g., attention, aggression, arousal dysregulation) that may be early mental health indicators.

\section{Electroconvulsive therapy during pregnancy}

Electroconvulsive therapy has long been regarded as a safe and effective treatment for severe depression in pregnancy, especially when the depressive disorder is life-threatening or fails to respond to antidepressant drugs. There is little evidence that it is harmful to the woman or fetus when both are carefully monitored [53].

\section{Diagnosing a depressive disorder in perinatal women}

Routine use of a self-report screening instrument does not supplant clinical diagnosis but can determine which women require further assessment. Many questionnaires include questions about fatigue, changes in sleep, or alterations in appetite that may be indicative of a depressive disorder but are also common in unaffected pregnant women. Either using a scale that has been validated among pregnant women or clarifying a woman's response to such questions can reduce false-positive rates.

The most commonly used screening questionnaire for depression in pregnancy is EPDS and includes 10 items [54]. A cutoff of at least 12 is often recommended in pregnancy [55]. The nine-item Patient Health Questionnaire is also useful and has been evaluated in an obstetrical-gynecological setting [56]. 


\section{Treating a pregnant woman who is depressed}

\subsection{Behavioral treatments for mood disorders}

Many patients with mild-to-moderate depression can be treated by psychosocial approaches including individual and group psychotherapy in lieu of medication. Patients with residual symptoms, those at high risk of relapse, those with comorbid conditions such as panic disorder and those who prefer to avoid medication may benefit from psychotherapy. This is an especially critical option for women preparing for conception or currently pregnant since a large percentage of women may plan to avoid medication.

Cognitive behavioral therapy (CBT) or interpersonal psychotherapy (IPT) have been shown to be effective for depression in pregnant women [57]. Web and computer-based cognitive behavioral therapy are options if there are few behavioral therapists in a patient's area or she has monetary limitations. While evidence for supportive and psychodynamic psychotherapy is limited, these approaches are also reasonable if IPT and CBT are unavailable.

\subsection{Antidepressant treatment during pregnancy}

There are no antidepressant drug efficacy trials in depressed pregnant women. However, there is little reason to think that response would differ between pregnant and nonpregnant women. Guidelines for treatment of depression and mania in pregnancy have been published [58-60].

It is ideal, but not always possible, to evaluate a woman with a past or current depressive illness prior to conception. In 2001, more than 6 million women conceived in the United States and nearly half of these were unintended [61]. Accordingly, this document presents algorithms for the management of a depressed woman who is contemplating pregnancy and is taking antidepressant medication (Fig. 1), pregnant and not receiving treatment for depression (Fig. 2) and pregnant but already undergoing pharmacological treatment for depression (Fig. 3). For all, healthy lifestyles, such as nutritional adequacy and weight management, regular prenatal care and childbirth education should be emphasized and supported. Treatment of addictions such as cigarettes, alcohol and other exposures is appropriate to reduce maternal-fetal risk.

Before prescribing any medication, document all drug and environmental exposures dating back to conception in the record. Careful documentation of a risk benefit discussion regarding medication or other treatment is imperative [59].

\subsection{Pre-conceptional patients}

When depressed patients present prior to conception for evaluation, her provider should determine whether the patient has moderate to severe symptoms (see algorithm in Fig. 1). If the evaluation is conducted by an obstetrical provider and the patient has suicidal or acute psychotic symptoms, she should be promptly referred to a psychiatrist for aggressive treatment. In such instances, it is judicious for the patient to wait a period of time after she has achieved euthymia before she attempts to conceive. Whether the patient has an evaluation with an obstetrician or a psychiatrist, if she has moderate to severe symptoms, her clinician should strongly consider continuation and optimization of her antidepressant medication. It is prudent to counsel the patient to wait for a period of time prior to conceiving in this instance as well. While it is difficult to specify an exact or optimal length of time for all patients, guidelines such as those from the Agency for Healthcare Quality and Research, suggest that antidepressant treatment for a first, acute episode of depression should endure at least 6-12 months [62]. 
Patients with mild or no symptoms for 6 months or longer may be candidates for medication taper and discontinuation prior to conception. The patient's psychiatrist and obstetrician can collaborate with the patient to make the decision regarding a hiatus from pharmacotherapy. Generally, if a woman is a candidate for stopping pharmacological treatment, slow taper such as $25 \%$ reduction in dose every 1-2 weeks with close monitoring for relapse or discontinuation symptoms is preferable.

Patients with a history of severe, recurrent major depressive disorders (MDD), psychosis, bipolar illness, psychiatric comorbidity that requires pharmacotherapy, or history of a serious suicide attempt may not be candidates for medication discontinuation. These individuals should continue psychotropic medication and her obstetrician and psychiatrist, if available, should coordinate her care so that she has optimal prophylaxis from psychiatric illness relapse but also appropriate obstetrical care. Some women may also benefit from referral to a therapist who can provide psychotherapy. While CBT or IPT are preferable, other types of counseling may be helpful if empiric-based therapies are not available.

\subsection{Pregnant patient who is not receiving pharmacotherapy}

It is common to diagnose untreated depression during pregnancy and to encounter patients who have discontinued their medications but are symptomatic. The algorithm presented in Fig. 2 summarizes recommendations for management of a depressed woman who is pregnant but not receiving treatment for her illness. Again, if an obstetrical provider encounters a patient with suicidal or psychotic symptoms, a psychiatrist who can optimize her pharmacotherapy should see the patient emergently. If possible, the psychiatrist should avoid first trimester use of antiepileptic agents, newer antipsychotic agents and antidepressants. The former are teratogens and are appropriate if alternatives are ineffective; there is less reproductive safety information about new (atypical) antipsychotics and antidepressants than agents that have been on the market for a decade or longer. An additional step is to assess whether the patient would consider pharmacotherapy. If a woman prefers not to take antidepressant medication and she is not gravely disabled or at high risk of relapse, she may benefit from psychotherapy. Psychiatrists should manage women who suffer from bipolar affective disorder since monotherapy with an antidepressant may trigger mania and psychosis. If the patient lives in a rural or other area where a psychiatrist is not available, the obstetrical provider may decide to seek expert consultation with regard to the management of the patient.

The treatment choice for other patients will depend upon the safety profile of the medications, the stage of gestation, the patient's symptoms, history and therapeutic preferences. The dose of agents metabolized primarily by cytochrome P450 2D6 or P450 3A4 may require an increase in the second half of pregnancy [63]. If a patient has an agitated depression, a more sedating antidepressant such as a TCA may be preferable. Mirtazepine is also sedating but there are few data on its use in pregnancy. TCAs and some SSRIs will also augment a patient's appetite if she has difficulty gaining weight. Women who are depressed and smoke cigarettes may benefit from treatment with bupropion, although a history of seizures or bulimia would preclude use of this agent.

\subsection{Patient with current or recent MDD who is taking antidepressants in pregnancy}

A woman may seek assistance about whether to continue or taper her antidepressant medication. An algorithm to address this issue is illustrated in Fig. 3. As above, a woman with evidence of current suicidal or psychotic thoughts will need to be managed aggressively by her psychiatrist. If the patient is psychiatrically stable and prefers continuation of medication, the psychiatric and obstetrical clinician should discuss risk/ benefit issues and document this discussion and the patient's choice, in her medical record. 
Respect for the patient's preferences is paramount since she will likely have a sense of her ability to manage a pregnancy off medication. If the patient is willing to consider discontinuation of medication and she is not currently having symptoms, then, depending upon the individual's psychiatric history, a trial of medication taper may be appropriate. Women who have a history of severe, recurrent depression, even if currently asymptomatic or minimally symptomatic, are at high risk of relapse if medication is discontinued. In one prospective cohort study of women who suffered from recurrent depression, the risk of relapse was sixfold higher if women elected to discontinue antidepressant treatment in pregnancy compared to women who continued treatment at the same dose throughout pregnancy [64].

Patients who have recurrent MDD or are symptomatic despite ongoing medication may benefit from psychotherapy that may either augment or replace pharmacotherapy. Patients who have not responded adequately to solo antidepressant treatment with a solid trial of psychotherapy, have severe illness with abrupt relapse after medication discontinuation or have a concurrent psychiatric condition that requires medication management, are candidates for continued antidepressant treatment.

Patients who are asymptomatic, have had a period of stability on treatment or have responded well in the past to psychotherapy may be candidates for medication taper and discontinuation with additional psychotherapy as needed. Their clinicians should monitor them for signs and symptoms of relapse.

\section{Frequently Asked Questions}

1. Should women who are being treated with paroxetine prior to conception switch to an alternative SSRI?

The decision to continue or change medication is a collaborative one between the physician and the patient, and there is no universal "best answer" for all women. The data that would link paroxetine use during the first trimester with cardiac malformations are not strong but it prompted the US Food and Drug Administration to reclassify the agent as a category " $D$ " drug. Nonetheless, some mothers may be concerned about media reports. If paroxetine is the only antidepressant that she has tried, substitution may be reasonable; however, substitution always carries the risk of relapse. If she has responded to paroxetine only after several other failed drug trials, substitution becomes difficult to justify. The clinician should review data regarding paroxetine as outlined in the preceding sections, document the discussion and the woman's questions carefully in the medical record.

2. Given the recently reported six-fold relative risk for persistent pulmonary hypertension of the newborn associated with SSRI exposures after 20 weeks gestation, how should pregnant women receiving SSRI therapy be counseled?

The six-fold risk is a relative risk; the absolute risk increases from 1 to 2 per 1000 $(0.1-0.2 \%)$ to $3-12$ per $1000(0.3-1.2 \%)$ with exposure to SSRI [44,49]. The risk for PPHN was increased for exposures to SSRI after 20 weeks of gestation and did not occur for non-SSRI antidepressants [44]. However, the risk of PPHN could be related to the duration of antidepressant use or other maternal illness severity characteristics [33]. Factors of illness history, risk of relapse with a change of agent and patient preference must be considered here as in Question 1 above.

3. In view of reports regarding neonatal SSRI effects, how should women who receive SSRI therapy in the third trimester be counseled? 
Third trimester use of TCAs and SSRIs have been associated with neonatal signs which are either due to the presence of antidepressants in the infant at birth (akin to side effects) or discontinuation effects. The majority of infants have signs that are mild and transient (most have disappeared by two weeks). Clinicians should consider preparing mothers for the possibility of infant effects such as restlessness and irritability. Such behaviors are not limited to SSRI exposed infants but are found in neonates exposed to TCAs as well as non-psychotropic exposure, including nicotine, as well as neonates with no exposures. Discontinuing medication to avoid symptoms in the neonate may lead to relapse in the mother.

4. What are the absolute contraindications to stopping SSRI therapy in pregnancy?

Women with severe depression characterized by suicide attempts, functional incapacitation or weight loss should be continued on their medication. The risk of recurrence or escalating symptoms is high after treatment discontinuation and can result in deterioration of the woman's physical health and possibly suicide. In the event that a patient refuses pharmacologic treatment, it is imperative for a psychiatrist to be part of the treatment team to reinstitute treatment if clinical deterioration occurs. Ideally, a plan for alternative treatment and monitoring of the patient's well being should be developed before medication discontinuation.

5. Are there pregnant patients for whom a TCA or bupropion would be a superior therapeutic option?

The antidepressant bupropion is also a therapy for nicotine addiction and may have added benefit for women who smoke and are depressed. However, not all patients respond to buproprion and clinicians should recognize that a change of medication may lead to relapse. This medication is contraindicated for individuals who have an eating disorder or seizure disorder. Tricyclic antidepressants are reasonable for use during pregnancy and an advantage is the ability of the clinician to monitor serum levels alongside response. However, anticholinergic side effects (e.g., dry mouth, constipation and sedation) and toxicity in overdose are disadvantages. As with bupropion, a patient who has responded to an SSRI may not respond to a TCA if she is switched to this medication.

6. Which pregnant patients are candidates for electroconvulsive therapy?

Patients who have not responded to antidepressant therapy or who are psychotic, suicidal or severely disabled are candidates for electroconvulsive therapy. Other patients with severe depression may prefer electroconvulsive therapy because of the rapidity of response to this modality.

\section{Acknowledgments}

The authors would like to thank Darrel Regier, M.D., M. P.H., Harold Goldstein, Ph.D., Stanley Zinberg, M.D., M. S., Nancy O'Reilly, MHS., Debra Hawks, M.P.H., and Kristin Edwards for administrative support. They would also like to acknowledge the assistance of Paul Sirovatka, M.S., who was an important member of the team that constructed this document.

\section{References}

1. Gaynes BN, et al. Perinatal depression: prevalence, screening accuracy and screening outcomes. Evid Rep/Technol Assess (Summ). 2005:1-8.

2. Cooper WO, Pont ME, Ray WA. Increasing use of antidepressants in pregnancy. Am J Obstet Gynecol. 2007; 196:544.e1. [PubMed: 17547888]

3. Kitamura T, Shima S, Sugawara M, Toda MA. Psychological and social correlates of the onset of affective disorders among pregnant women. Psychol Med. 1993; 23:967-975. [PubMed: 8134520] 
4. Swallow BL, Lindow SW, Masson EA, Hay DM. Psychological health in early pregnancy: relationship with nausea and vomiting. J Obstet Gynaecol. 2004; 24:28-32. [PubMed: 14675977]

5. Sugiura-Ogasawara M, et al. Depression as a potential causal factor in subsequent miscarriage in recurrent spontaneous aborters. Hum Reprod. 2002; 17:2580-2584. [PubMed: 12351532]

6. Nakano Y, et al. Psychosocial predictors of successful delivery after unexplained recurrent spontaneous abortions: a cohort study. Acta Psychiatr Scand. 2004; 109:440-446. [PubMed: 15117289]

7. Nelson BA, McMahon K, Joffe M, Brensinger C. The effect of depressive symptoms and optimism on the risk of spontaneous abortion among innercity women. J Womens Health. 2003; 12:569-576.

8. Steer RA, Scholl TO, Hediger ML, Fischer RL. Self-reported depression and negative pregnancy outcomes. J Clin Epidemiol. 1992; 45(10):1093-1099. [PubMed: 1474405]

9. Oberlander T, Warburton W, Misri S, Aghajanian J, Hertzman C. Neonatal outcomes after prenatal exposure to selective serotonin reuptake inhibitor antidepressants and maternal depression using population-based linked health data. Arch Gen Psychiatry. 2006; 63:898-906. [PubMed: 16894066]

10. Andersson L, Sundstrom-Poromaa I, Wulff M, Astrom M, Bixo M. Neonatal outcome following maternal antenatal depression and anxiety: a population-based study. Am J Epidemiol. 2004; 159:872-881. [PubMed: 15105180]

11. Suri R, et al. Effects of antenatal depression and antidepressant treatment on gestational age at birth and risk of preterm birth. Am J Psychiatry. 2007; 164:1206-1213. [PubMed: 17671283]

12. Orr S, James S, Blackmore Prince C. Maternal prenatal depressive symptoms and spontaneous preterm births among African-American women in Baltimore, MD. Am J Epidemiol. 2002; 156:797-802. [PubMed: 12396996]

13. Field T, Diego M, Hernandez-Reif M. Prenatal depression effects on the fetus and newborn: a review. Infant Behav Dev. 2006; 29:445-455. [PubMed: 17138297]

14. DiPietro J, et al. Maternal psychological distress during pregnancy in relation to child development at age two. Child Dev. 2006; 77:573-587. [PubMed: 16686789]

15. Punamaki R. Maternal mental health and medical predictors of infant developmental and health problems from pregnancy to one year: does former infertility matter? Infant Behavior \& Development. 2006; 29:230-242. [PubMed: 17138278]

16. Deave T, Heron J, Evans J, Emond A. The impact of maternal depression in pregnancy on early child development. BJOG. 2008; 115:1043-1051. [PubMed: 18651886]

17. Headley J, Northstone K, Simmons H, Golding J. ALSPAC Study Team. Medication use during pregnancy: data from the Avon Longitudinal Study of Parents and Children. Eur J Clin Pharmacol. 2004; 60:355-661. [PubMed: 15168103]

18. Källén BA, Otterblad Olausson P. Maternal use of selective serotonin re-uptake inhibitors in early pregnancy and infant congenital malformations. Birth Defects Res A Clin Mol Teratol. 2007; 79:301-308. [PubMed: 17216624]

19. Chambers CD, Johnson KA, Dick LM, Felix RJ. Birth outcomes in pregnant women taking fluoxetine. N Engl J Med. 1996; 335:1010-1015. [PubMed: 8793924]

20. Chun-Fai-Chan B, et al. Pregnancy outcome of women exposed to bupropion during pregnancy: a prospective comparative study. Am J Obstet Gynecol. 2005; 192:932-936. [PubMed: 15746694]

21. Einarson A. Pregnancy outcome following gestational exposure to venlafaxine: A multicenter prospective controlled study. Am J Psychiatry. 2001:158.

22. Einarson A, et al. A multicentre prospective controlled study to determine the safety of trazodone and nefazodone use during pregnancy. Can J Psychiatry. 2003; 48:106-110. [PubMed: 12655908]

23. Kulin N, et al. Pregnancy outcome following maternal use of the new selective serotonin reuptake inhibitors. JAMA. 1998; 279(8):609-610. [PubMed: 9486756]

24. Pastuszak A, et al. Pregnancy outcome following first-trimester exposure to fluoxetine (Prozac). JAMA. 1993; 269(17):2246-2248. [PubMed: 8474204]

25. Hemels M, Einarson A, Koren G, Lanctot K, Einarson T. Antidepressant use during pregnancy and the rates of spontaneous abortions: a meta-analysis. Ann Pharmacother. 2005; 39:803-809.

[PubMed: 15784808] 
26. Saraiya M, Berg C, Shulman H, Green C, Atrash H. Estimates of the Annual Number of Clinically Recognized Pregnancies in the United States, 1981-1991. Am J Epidemiol. 1999; 149:1025-1029. [PubMed: 10355378]

27. Simon G, Cunningham M, Davis R. Outcomes of prenatal antidepressant exposure. Am J Psychiatry. 2002; 159:2055-2061. [PubMed: 12450956]

28. Wen S, et al. Selective serotonin reuptake inhibitors and adverse pregnancy outcomes. J Obstet Gynecol. 2006; 194:961.

29. Djulus J, et al. Exposure to mirtazapine during pregnancy: a prospective, comparative study of birth outcomes. J Clin Psychiatry. 2006; 67:1280-1284. [PubMed: 16965209]

30. Sivojelezova A, Shuhaiber S, Sarkissian L, Einarson A, Koren G. Citalopram use in pregnancy: prospective comparative evaluation of pregnancy and fetal outcome. J Obstet Gynecol. 2005; 193:2004.

31. Malm H, Klaukka T, Neuvonen P. Risks associated with selective serotonin reuptake inhibitors in pregnancy. Obstet Gynecol. 2005; 106:1289-1296. [PubMed: 16319254]

32. Kallen B. Neonate characteristics after maternal use of antidepressants in late pregnancy. [see comment]. Arch Pediatr Adolesc Med. 2004; 158:312-316. [PubMed: 15066868]

33. Oberlander T, Warburton W, Misri S, Aghajanian J, Hertzman C. Effects of timing and duration of gestational exposure to serotonin reuptake inhibitors: population based study. Br J Psychiatry. 2008; 192:338-343. [PubMed: 18450656]

34. Hamilton B, Martin J, Ventura S. Births: preliminary data for 2005. National vital statistics report. National Center for Health Statistics. 2007

35. Costei A, Kozer E, Ho T, Ito S, Koren G. Perinatal outcome following third trimester exposure to paroxetine. Arch Pediatr Adolesc Med. 2002; 156:1129-1132. [PubMed: 12413342]

36. Altshuler L, et al. Pharmacologic management of psychiatric illness during pregnancy: dilemmas and guidelines. [see comments]. Am J Psychiatry. 1996; 153:592-606. [PubMed: 8615404]

37. Louik C, Lin A, Werler M, Hernandez-Diaz S, Mitchell A. First-trimester use of selective serotonin-reuptake inhibitors and the risk of birth defects. N Engl J Med. 2007; 356:2675-2683. [PubMed: 17596601]

38. Alwan S, et al. Use of selective serotonin-reuptake inhibitors in pregnancy and the risk of birth defects. N Engl J Med. 2007; 356:2684-2692. [PubMed: 17596602]

39. Kallen B, Otterblad Olausson P. Antidepressant drugs during pregnancy and infant congenital heart defect. Reprod Toxicol. 2006; 21:221. [PubMed: 16406480]

40. Cole J, et al. Bupropion in pregnancy and the prevalence of congenital malformations. Pharmacoepidemiol Drug Saf. 2007; 16:474-484. [PubMed: 16897811]

41. Einarson A, et al. Evaluation of the risk of congenital cardiovascular defects associated with use of paroxetine during pregnancy. Am J Psychiatry. 2008; 165:749-752. [PubMed: 18381907]

42. Oberlander TF. Major congenital malformations following prenatal exposure to serotonin reuptake inhibitors and benzodiazepines using population-based health data. Birth Defects Res B Dev Reprod Toxicol. 2008; 83:68-76. [PubMed: 18293409]

43. Lennestal R, Kallen B. Delivery outcome in relation to maternal use of some recently introduced antidepressants. J Clin Psychopharmacol. 2007; 27:607-613. [PubMed: 18004128]

44. Chambers $\mathrm{C}$, et al. Selective serotonin-reuptake inhibitors and risk of persisitent pulmonary hypertension of the newborn. N Engl J Med. 2006; 354:579-587. [PubMed: 16467545]

45. Oberlander T, et al. Infant serotonin transporter (SLC6A4) promoter genotype is associated with adverse neonatal outcomes after prenatal exposure to serotonin reuptake inhibitor medications. Mol Psychiatry. 2008; 13:65-73. [PubMed: 17519929]

46. Laine K, Heikkinen T, Ekblad U, Kero P. Effects of exposure to selective serotonin reuptake inhibitors during pregnancy on serotonergic symptoms in newborns and cord blood monoamine and prolactin concentrations. Arch Gen Psychiatry. 2003; 60:720-726. [PubMed: 12860776]

47. Oberlander T, et al. Pharmacologic factors associated with transient neonatal symptoms following prental psychotropic medication exposure. J Clin Psychiatry. 2004; 65:230-237. [PubMed: 15003078] 
48. Zeskind P, Stephens L. Maternal selective serotonin reuptake inhibitor use during pregnancy and newborn neurobehavior. Pediatrics. 2004; 113:368-375. [PubMed: 14754951]

49. Källén B, Olausson P. Maternal use of selective serotonin re-uptake inhibitors and persistent pulmonary hypertension of the newborn. Pharmacoepidemiol Drug Saf. 2008; 17:801-806. [PubMed: 18314924]

50. Greenough A, Khetriwal B. Pulmonary hypertension in the newborn. Paediatr Respir Rev. 2005; 6:111-116. [PubMed: 15911456]

51. Nulman I, et al. Neurodevelopment of children exposed in utero to antidepressant drugs. N Engl J Med. 1997; 336:258-262. [PubMed: 8995088]

52. Nulman I, et al. Child development following exposure to tricyclic antidepressants or fluoxetine throughout fetal life: a prospective, controlled study. Am J Psychiatry. 2002; 159:1889-1895. [PubMed: 12411224]

53. Miller L. Use of electroconvulsive therapy during pregnancy. Hosp Commun Psychiatry. 1994; 45(5):444-450.

54. Cox, J.; Holden, J. Use and misuse of the Edinburgh Postnatal Depression Scale. Glasgow: The Royal College of Psychiatrists; 1994. Perinatal psychiatry.

55. Matthey S, Henshaw C, Elliott S, Barnett B. Variability in use of cut-off scores and formats on the Edinburgh Postnatal Depression Scale: implications for clinical and research practice. Arch Women Ment Health. 2006; 9:309-315.

56. Spitzer R, Williams J, Kroenke K, Hornyak R, McMurray J. Validity and utility of the Patient Health Questionnaire (PHQ) in assessing 3000 obstetric gynecology patients: The Prime-MD PHQ obstetric gynecology study. Am J Obstet Gynecol. 2000; 183(3):759-769. [PubMed: 10992206]

57. Spinelli M, Endicott J. Controlled clinical trial of interpersonal psychotherapy versus parenting education program for depressed pregnant women. Am J Psychiatry. 2003; 160:555-562. [PubMed: 12611838]

58. Altshuler LL, et al. The Expert Consensus Guideline Series. Treatment of depression in women. Postgrad Med. 2001:1-107. [PubMed: 11500997]

59. Wisner K, Gelenberg A, Leonard H, Zarin D, Frank E. Pharmacologic treatment of depression during pregnancy. JAMA. 1999; 282:1264-1269. [PubMed: 10517430]

60. Yonkers K, et al. Management of bipolar disorder during pregnancy and the postpartum period. Am J Psychiatry. 2004; 161:608-620. [PubMed: 15056503]

61. Finer L, Henshaw S. Disparities in rates of unintended pregnancy in the United States, 1994 and 2001. Perspect Sex Reprod Health. 2006; 38:90-96. [PubMed: 16772190]

62. Rush, A., et al. Clinical Practice Guideline Number. 5th ed.. Rockville, MD: Agency for Health Care Policy and Research; 1993. Depression in primary care: Vol. 2. Treatment of Major Depression.

63. Wisner K, Perel J, Wheeler S. Tricyclic dose requirements across pregnancy. Am J Psychiatry. 1993; 150(10):1541-1542. [PubMed: 8379562]

64. Cohen L, et al. Relapse of major depressive during pregnancy in women who maintain or discontinue antidepressant treatment. JAMA. 2006; 295:499-507. [PubMed: 16449615] 


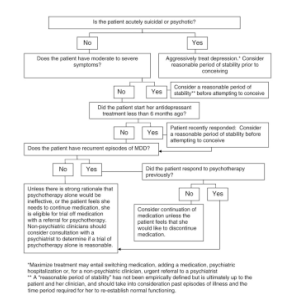

Fig. 1.

Patient is contemplating pregnancy and is undergoing pharmacological treatment for depression. 


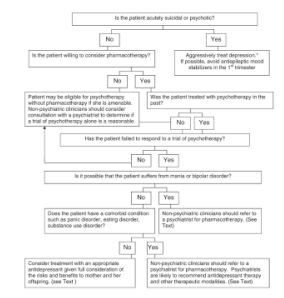

Fig. 2.

Patient is in episode of MDD, is pregnant and is not taking antidepressants. 


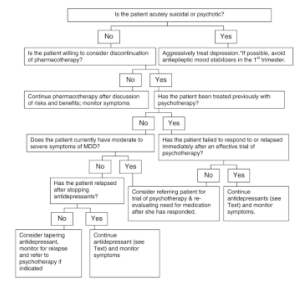

Fig. 3.

Patient with MDD who is pregnant and currently taking antidepressants. 